\title{
Damage and stress evolution in the bondlines of metallic adhesively bonded joints accompanied by bondline thickness effect
}

\author{
Wei Xu*,1, Huichen Yu, Chunhu Tao \\ Beijing Key Laboratory of Aeronautical Materials Testing and Evaluation, Science and Technology on Advanced High Temperature Structural Materials \\ Laboratory, Beijing Institute of Aeronautical Materials, Beijing 100095, PR China
}

\section{A R T I C L E I N F O}

Article history:

Accepted 13 February 2015

Available online 24 February 2015

Keywords:

Finite element analysis

Damage evolution

Single lap joint

Cohesive interface model

Cohesive length scale

\begin{abstract}
A B S T R A C T
Metallic adhesively bonded joints have a wide range of applications in engineering fields. The damage and the stress evolution in the bondlines of the joints would occur simultaneously when the joints are subjected to external loads. In the present research, the influence of the bondline thickness on the damage and stress evolution of metallic adhesive bonding structures are investigated, with the cohesive interface model employed to equivalently simulate the bondline with various thicknesses. A prediction approach is employed to determine the cohesive parameters for the bondline with the thickness varied. Then a numerical example is presented to explore the bondline thickness-dependence strength and stress distribution in the bondline, followed by the validation with the existing experimental and theoretical results. Furthermore, as the main part of this paper, the influences of the bondline thickness on the damage and stress evolution in the bondline are investigated, involving the situations of the extremities and the whole bondline. The results show that, no matter in the extremities or in the whole bondline, the damage and stress evolutions are mutually influential processes, both of which are affected by the bondline thickness significantly.
\end{abstract}

(c) 2015 Elsevier Ltd. All rights reserved.

\section{Introduction}

Modern engineering structures have an increasing demand for the higher strength/weight ratio, especially in the fields of automobile, aeronautics and astronautic industries. Thus it is significant to use the novel lightweight materials with high strength and new joining techniques. Adhesive bonding is such an advanced joining technique with great potential for lightweight constructions. Compared with traditional mechanical assembly technologies (e.g., bolted, pinned, or riveted methods), it has a lot of advantages, such as reducing the stress concentration, wide applicability and lightweight. Thus adhesively bonded joints have been increasingly used in many engineering fields.

The ultimate failure strength prediction of adhesively bonded joints is one of the most important issues in this field, since the failure strength is a key parameter for design and health evaluation of adhesively bonded structures. Thus some methods are hence developed in order to predict the failure strength when the material parameters of adhesives are predefined. The overall failure strength prediction usually involves two main approaches: the one method to predict the overall strength is to assess the stress distribution in the

\footnotetext{
* Corresponding author. Tel.: +86 10 62496728; fax: +86 1062496733.

E-mail address: xuwei@lnm.imech.ac.cn (W. Xu).

${ }^{1}$ Former affiliation: Institute of Mechanics, Chinese Academy of Sciences, Beijing, PR China.
}

bondline, either by numerical models or by analytical methods [1-4]. Most of the adhesively bonded joints would lose their load bearing capacity due to the failure of the bondlines, thus the accurate determination of the stress fields in the bondline is the first step to the precise prediction of the failure load of an adhesive joint. The stress corresponding to the critical load when the bondline stress reaches its admissible value is defined as the overall strength. Some failure or strength criteria are usually employed in this approach $[5,6]$, such as the von Mises stress criterion and the principal stress criterion. Other researchers [7] adopted a twofold criterion involving stress and energy conditions simultaneously to predict the failure loads of adhesive joints subjected to diverse loadings. By contrast, the other method to predict the overall strength is a direct one, which is to obtain the load-displacement curves by simulating the loading process of adhesively bonded joints. Then the overall strength or the failure load can be obtained directly.

The first prediction method needs to assess the local stresses in some significant positions such as the extremities of the bondline, instead of the whole bondline, since the stress concentration of the extremities is much noticeable. On the contrary, the latter method for the overall mechanical behaviors is a global method [8], which neglects the stress singularity in the bondline, and it merely concerns the overall mechanical response of the structures subjected to external loads. The peak load could not be always treated as the admissible load because the irreversible damage has occurred in the bondline before the peak load is reached. Thus the 
overall mechanical behaviors should be obtained considering the local damage and stress distribution meanwhile.

Among all factors, the bondline thickness is one of most significant geometrical parameters affecting the overall strength of bonding structure. Thus the investigations on the influence of bondline thickness have been carried out by so many researchers, including the experimental studies $[9,10]$ and the modeling studies [11-13]. Most of the experimental results have indicated that the overall strength of the bonding structures definitely depends on the bondline thickness. Recently, some modeling prediction methods have been established to obtain the computational results compared with the experiments. Moradi et al. [14] presented the influence of the bondline thickness on the failure load of joints by employing the stress and energy-based criteria together with a matched asymptotic procedure. Also the couple stress and energy criterion was employed by other researchers $[5,15]$ to consider the influence of bondline thickness. Some failure load models were usually employed in their investigations. For example, the maximum principal stress criterion was used in Ref. [5] to determine the failure load of joints. Compared with the aforementioned researches, cohesive zone model (CZM) can be regarded as a direct approach, which has been proved to be successful in this topic. The significant advantage of employing cohesive zone model is that the overall mechanical behaviors (i.e., load-displacement curves) of joints can be directly obtained. And the effect of bondline thickness on the overall mechanical behaviors can be clearly presented. Thus the strength (or failure load) of the joints can be determined directly and easily just as the same way as the experimental approaches. The other advantage of CZM is that damage evolution of the interface or bondline could be captured as well. However, the tough challenge of employing CZM in this topic is how to assign the values of the cohesive parameters for various bondline thicknesses. Some good jobs have been done for predicting the overall mechanical strength of bonding structures with various bondline thicknesses. However, the understanding to the mechanisms of thickness-dependence cohesive properties has been still local. Besides, the local and overall stress analysis and damage evolution in the bondline with various thicknesses are lacking as well.

In the present research, a numerical model utilizing finite elements method (FEM) is established to describe the mechanical behavior of the metallic single lap joint subjected to a tensile load. A cohesive interface model considering the damage evolution is employed to simulate the bondline, with the cohesive parameters obtained via the previously proposed thickness-dependence prediction method. The present paper focuses on the mutual influences between the damage evolution and stress distribution in the bondline with various thicknesses. Finally, the effect of the bondline thickness on the accuracy of the ultimate failure strength assessment would be discussed based on the cohesive length scale.

\section{Cohesive interface model}

\subsection{Bilinear cohesive zone model}

Cohesive zone models (CZMs) based on traction-separation (i.e., $\mathrm{T}-\mathrm{S}$ for short) laws are well suitable to describe the de-cohesion behavior in composite structures. The CZMs require T-S relations for characterizing their constitutive laws. So far, considerable researches have focused on the constitutive laws of CZMs and their applications [16,17]. It has been established that whilst the peak value and area of the T-S curve are vital for capturing the interface separation behavior, its precise shape is of less significance [18]. Consequently, for simplicity, the bilinear T-S law shown in Fig. 1 is selected for the present study. To distinguish the normal T-S law from the shear one, the superscript " $n$ " represents the normal

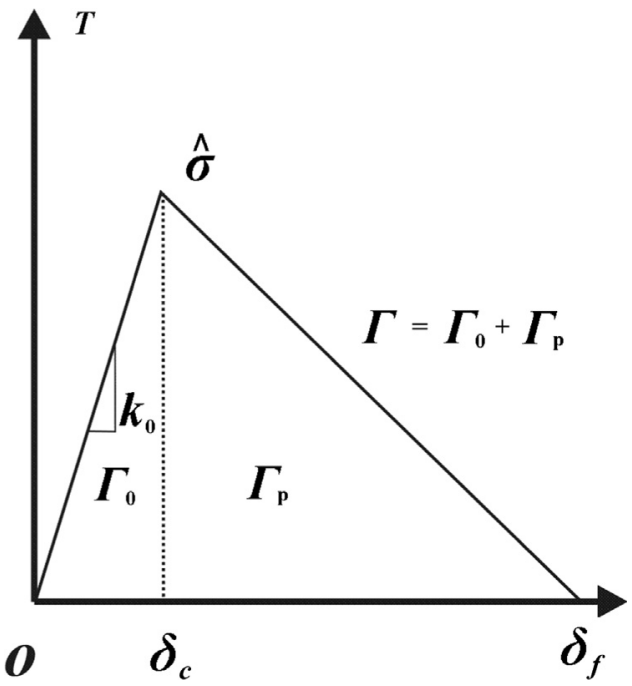

Fig. 1. Typical bilinear traction-separation law of cohesive zone model (the superscripts $\mathrm{n}$ and $\mathrm{s}$ denoting the normal and shear directions respectively, are omitted).

direction and "s" denotes the shear direction, which are omitted in Fig. 1 for simplicity, $\delta_{c}$ and $\delta_{f}$ are the critical and failure separation displacement, respectively, and $T$ is the traction stress.

Since the maximum value of $T^{\mathrm{n}}$ is $\hat{\sigma}^{\mathrm{n}}$ while that of $\left|T^{\mathrm{s}}\right|$ is $\hat{\sigma}^{\mathrm{s}}$, the fracture energy in the two directions can be expressed as

$$
\begin{gathered}
\Gamma^{\mathrm{n}}=\int_{0}^{\delta_{f}^{\mathrm{n}}} T^{\mathrm{n}} d \delta^{\mathrm{n}}=\frac{1}{2} \hat{\sigma}^{\mathrm{n}} \delta_{f}^{\mathrm{n}} \\
\Gamma^{\mathrm{s}}=\int_{0}^{\delta_{f}^{\mathrm{s}}} T^{\mathrm{s}} d \delta^{\mathrm{s}}=\frac{1}{2} \hat{\sigma}^{\mathrm{s}} \delta_{f}^{\mathrm{s}}
\end{gathered}
$$

As the loading is increased beyond a critical value, the cohesive layer begins to soften, and degrade, namely, the cohesive layer is now in the damaged (or softening) state. Typically, damage is initiated when a certain criterion is satisfied. In the present study, inspired by the bilinear law of Fig. 1, the quadratic nominal stress criterion is adopted to characterize interfacial damage, described as

$\left(\frac{<T^{\mathrm{n}}>}{\hat{\sigma}^{\mathrm{n}}}\right)^{2}+\left(\frac{T^{\mathrm{s}}}{\hat{\sigma}^{\mathrm{s}}}\right)^{2}=1$

where $<>$ represents the Macaulay bracket defined by $\langle x\rangle=$ $(x+|x|) / 2$, with the usual interpretation that a pure compressive deformation or stress state does not initiate damage. The peak traction stress components $\hat{\sigma}^{\mathrm{n}}$ and $\hat{\sigma}^{\mathrm{s}}$ are termed the normal and shear separation strengths, respectively.

\subsection{Description of damage evolution}

Damage variables describing the extent of damage in cohesive layer have a meaning physically equivalent to that introduced in the continuum damage mechanics (CDM) for engineering materials. In CDM, $D$ is linearly proportional to the ratio of current Young's stiffness $E$ of the material to its initial value $E_{0}$, i.e., $D=1-E / E_{0}$, if the damage is isotropic; for anisotropic damage, a tensor $\mathbf{D}$ is typically used [19].

For the two-dimensional problem employing the cohesive zone model, if the external loading process is mode-independent, or the deformation of cohesive layer is pure normal or shear mode, the damage of cohesive layer would occurs when the traction stress declines after its peak value is reached, and the damage variable 
can be expressed as,

$D=\frac{\delta_{f}\left(\delta-\delta_{c}\right)}{\delta\left(\delta_{f}-\delta_{c}\right)} \quad\left(\delta_{c} \leq \delta<\delta_{f}\right)$

Note that the superscripts denoting the normal and shear directions (i.e., $\mathrm{n}$ and $\mathrm{s}$ ) are also omitted for simplicity, followed by the equations below.

With reference to Fig. 1 , the stiffness in the softening state, $k_{d}$ decreases during damage evolution, which can be linked to the damage variable by

$k_{d}=(1-D) k_{0}$

Here, $k_{0}$ is the initial stiffness with the following relation with the separation stress,

$k_{0}=\frac{\hat{\sigma}}{\delta_{c}}$

It is well-known that the fracture in the bondline of a single lap joint is a mixed mode one, which means the failure behaviors in both normal and shear directions should be taken into account. Thus the cohesive zone model used here is also a mixed-mode one, with both shear and normal stress components considered simultaneously. It is assumed that damage occurs when Eq. (2) is satisfied and a single damage variable $D$ based on the total displacement jump $\Delta$ is introduced (i.e., $\Delta=\sqrt{<\delta^{\mathrm{n}}>^{2}+\left(\delta^{\mathrm{s}}\right)^{2}}$ ) [20,21], as

$D= \begin{cases}0 & \left(\Delta_{m} \leq \Delta_{c}\right) \\ \frac{\Delta_{f}\left(\Delta_{m}-\Delta_{c}\right)}{\Delta_{m}\left(\Delta_{f}-\Delta_{c}\right)} & \left(\Delta_{c}<\Delta_{m}<\Delta_{f}\right) \\ 1 & \left(\Delta_{m} \geq \Delta_{f}\right)\end{cases}$

where $\Delta_{c}$ and $\Delta_{f}$ denote the total displacement at damage initiation and complete failure. The quantity, $\Delta_{f}$ is determined by $\Delta_{f}=$ $2 \Gamma^{\text {mixed }} / T_{c}^{\text {eff }}$ with $T_{c}^{\text {eff }}$ denoting the effective traction at damage initiation (i.e., $T_{c}^{\text {eff }}=\sqrt{\left(T_{c}^{n}\right)^{2}+\left(T_{c}^{s}\right)^{2}}$ ). In Eq. (6), $\Delta_{m}$ denotes the maximum total displacement experienced during the loading history. The value of $D$ increases monotonically from 0 (corresponding to damage initiation) to 1 (corresponding to complete failure).

$\Gamma^{\text {mixed }}$ is the mixed total fracture energy of the adhesive. Generally, $\Gamma^{\text {mixed }}$ depends on the mode-mixity. In other words, $\Gamma^{\text {mixed }}$ varies as a function of the mode-mixity. In the present investigation, $\Gamma^{\text {mixed }}$ is determined by the linear fracture criterion, which can be expressed as

$\frac{G^{\mathrm{n}}}{\Gamma^{\mathrm{n}}}+\frac{G^{\mathrm{s}}}{\Gamma^{\mathrm{s}}}=1$

where $G^{\mathrm{n}}$ and $G^{\mathrm{s}}$ denote work done by the traction and its conjugate relative displacement in the normal and shear directions, respectively. $\Gamma^{\mathrm{n}}$ and $\Gamma^{\mathrm{s}}$ refer to the total energy required to cause failure in the pure normal and shear directions, respectively, as defined in Eq. (1). Consequently, $\Gamma^{\text {mixed }}$ can be obtained by,

$\Gamma^{\text {mixed }}=G^{\mathrm{n}}+G^{\mathrm{s}}$

when Eq. (7) is satisfied.

\subsection{Thickness-dependence cohesive parameters}

When the cohesive zone model is employed to simulate the bondline, the progressive failure of the adhesive could be captured by the aforementioned bilinear traction-separation law, which is defined by three key cohesive parameters, namely, initial stiffness, total fracture energy and separation strength. Since the cohesive modeling is an equivalent method, all of these cohesive parameters would be influenced by the bondline thickness, although the intrinsic material properties of the adhesive are unchangeable. The influences of the bondline thickness on the cohesive properties have been investigated by some researchers experimentally [10] and theoretically [12]. And some valuable results have been hence obtained. Among them, our previously proposed prediction model [12] has gained a certain success, which accurately predicts the relation between the bondline thickness and cohesive parameters. Later, some other researchers also adopted this theoretical model to address similar issues and obtained good results [11]. In this section, the prediction model is reviewed briefly as follows.

The initial stiffness of $\mathrm{CZ}$ model represents the slope value of the rising part of the T-S curve shown in Fig. 1. The initial stiffness is used to describe the ratio between the cohesive stress and separation displacement before the adhesive damage occurs, which is similar to the stiffness coefficient of a spring. Obviously, the initial stiffness is dominated by the elastic properties together with the bondline thickness $t$. In the present investigation, following the way in the previous researches [22], initial stiffness could be expressed as

$k_{0}=\frac{E}{t}$

where $E$ denotes the elastic modulus (involving Young's modulus and shear modulus components). Linked with the initial stiffness, the relation between the separation strength $\hat{\sigma}$ and critical separation displacement $k_{0}$ could be obtained,

$\delta_{c}=\frac{\hat{\sigma}}{k_{0}}$

Generally, the mixed fracture energy of CZM could be determined by Eq. (8) when considering the bondline as an equivalent cohesive layer. In other aspect, the bondline with a certain thickness would dissipate two types of energies, including the intrinsic cohesive energy $\Gamma_{o}$ and plastic dissipation energy $\Gamma_{p}$, which denote the energy making the bondline separated and the energy dissipated during the plastic deformation, respectively. $\Gamma_{o}$ could be regarded as intrinsic work of fracture associated with the embedded cohesive zone, and $\Gamma_{p}$ could be regarded as the contribution to the bond toughness arising from the plastic dissipation and stored elastic energy within the bondline [23,24]. In our previously proposed model, the total fracture energy $\Gamma$ can be expressed below:

$\Gamma=\Gamma_{0}+\Gamma_{p}= \begin{cases}\Gamma_{0}+U t & \left(t<2 r_{p}^{\max }\right) \\ \Gamma_{0}+2 U r_{p}^{\max } & \left(t \geq 2 r_{p}^{\max }\right)\end{cases}$

where $r_{p}^{\max }$ is maximum value of plastic zone height in front of crack tip, with the crack plane assumed in the middle of bondline, and $U$ is the area below the stress-strain curve of the tensile specimen for the adhesive material, also could be seen as the mean plastic work per unit adhesive volume [9].

Here, a dimensionless parameter $\eta$ is introduced based on intrinsic energy parameters, with the expression of,

$\eta=\frac{U t_{c}}{\Gamma_{0}}$

where $t_{c}$ denotes the minimum bondline thickness for which the separation strength $\hat{\sigma}$ would be identical to the bulk fracture strength $\sigma_{f}$. The critical thickness $t_{c}$ is usually determined by the plastic zone height $r_{p}^{\max }[12,23]$. Accordingly, the separation strength $\hat{\sigma}$ could be expressed in the dimensionless form in terms of the adhesive bulk strength $\sigma_{f}$, as depicted in Eq. (13).

$\frac{\hat{\sigma}}{\sigma_{f}}= \begin{cases}\sqrt{\frac{1+\eta\left(\frac{t}{t_{c}}\right)}{(1+\eta)\left(\frac{t}{t_{c}}\right)}} & \left(t<t_{c}\right) \\ 1 & \left(t \geq t_{c}\right)\end{cases}$ 

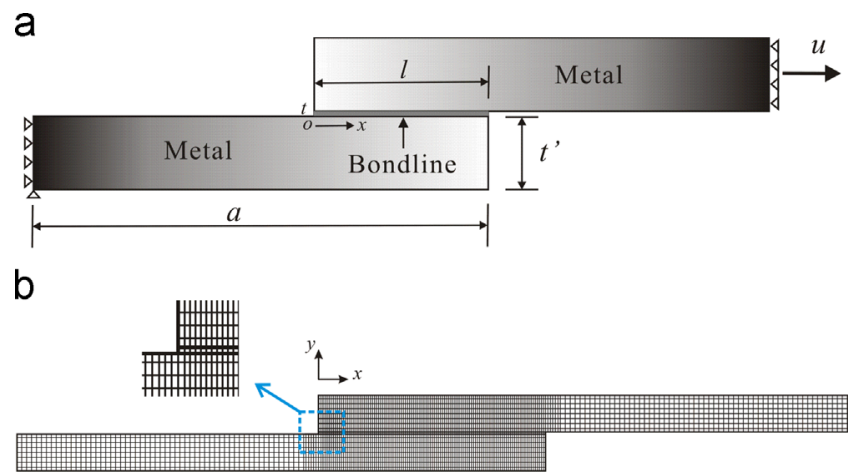

Fig. 2. (a) Numerical model of metallic single lap joint (SLJ) and (b) its finite element mesh.

Furthermore, total fracture energy $\Gamma$ can be further simplified into dimensionless form,

$\frac{\Gamma}{\Gamma_{0}}=\left\{\begin{array}{lc}1+\eta\left(\frac{t}{t_{c}}\right) & \left(t<t_{c}\right) \\ 1+\eta & \left(t \geq t_{c}\right)\end{array}\right.$

The detailed derivation of the approach for the thicknessdependence cohesive parameters is omitted here for simplicity, it could be found in our previous publication [12]. Furthermore, it should be pointed out the thickness-dependence prediction model is proposed based on the small-scale yield assumption. Thus the joints with the metallic adherends susceptible to plastic deformation (i.e., too soft or too thin) are not applicable with the present prediction model.

\section{Computational model}

\subsection{Modeling approach}

In this section, a numerical model of the metallic single lap joint (SLJ) is built with the commercially available FEM code ABAQUS. Since the width of the adherends used for the joint is far larger than their thickness, adhesive joints under tension can be simplified as the elastic-plastic plane strain problems. Among the adhesively bonded joints, the configuration of single lap joint is one of the most common joints. Due to the simplicity of the geometry, the single lap joint is widely used in many industry fields. Nevertheless, the stress distribution and fracture model in the bondline of SLJ are so complex. Especially, both cohesive properties in shear and normal directions contribute to the fracture behavior in bondline, thus the fracture mode of SLJ is so-called mixed mode. Fig. 2a depicts the computational model of the single lap joint, which consists of two same metallic adherends with the thickness of $h$, having a typical value of $2 \mathrm{~mm}$. The length of the adherends $a$, is assigned the value of $120 \mathrm{~mm}$. The adherends are connected by the bondline with the length of $l$, which is also called overlap length, assigned the value of $25 \mathrm{~mm}$. The adherends are meshed using fournode quadrilateral plane strain elements, of which the total number is set as 4450 upon checking the convergence of the numerical results. Subjected to the uniaxial tensile loading, the joint is taken to deform under plane strain. In the numerical models, the left side of the joint is fixed in horizontal direction, and the lower left corner is also fixed in the vertical direction. The model is loaded by the means of increasing displacement, and a uniform displacement of $u$ is applied to the right side of the joint.

The metallic adherends are modeled as elastic-plastic solids, with their true stress-strain curves fitted using power-law
Table 1

Material parameters for the adhesive [9].

\begin{tabular}{lc}
\hline Parameters & Hysol EA 9321 \\
\hline Young's modulus $E(\mathrm{GPa})$ & 3.87 \\
Poisson's ratio $\nu$ & 0.36 \\
Yield strength $\sigma_{s}(\mathrm{MPa})$ & 21.99 \\
Tensile strength $\sigma_{f}(\mathrm{MPa})$ & 45.97 \\
Toughness $U(\mathrm{MPa})$ & 1.16 \\
$\Gamma_{0}^{\mathrm{n}}\left(\mathrm{N} \mathrm{mm} \mathrm{mm}^{-1}\right)$ & 0.45 \\
$\Gamma_{0}^{\mathrm{s}}\left(\mathrm{N} \mathrm{mm}^{-1}\right)$ & 0.90 \\
\hline
\end{tabular}

Table 2

Thickness-dependence cohesive parameters of the bondline.

\begin{tabular}{llll}
\hline Bondline thickness $(\mathrm{mm})$ & 0.1 & 0.5 & 1.0 \\
\hline$k_{0}^{\mathrm{n}}\left(\mathrm{MPa} \mathrm{mm}^{-1}\right)$ & 38,700 & 7740 & 3870 \\
$k_{0}^{\mathrm{s}}\left(\mathrm{MPa} \mathrm{mm}^{-1}\right)$ & 14,200 & 2840 & 1420 \\
$\Gamma^{\mathrm{n}}\left(\mathrm{N} \mathrm{mm} \mathrm{mm}^{-1}\right)$ & 0.57 & 1.03 & 1.61 \\
$\left.\Gamma^{\mathrm{s}}(\mathrm{N} \mathrm{mm})^{-1}\right)$ & 1.02 & 1.48 & 2.06 \\
$\hat{\sigma}^{\mathrm{n}}(\mathrm{MPa})$ & 86.19 & 52.00 & 45.97 \\
$\hat{\sigma}^{\mathrm{s}}(\mathrm{MPa})$ & 29.14 & 15.73 & 13.12 \\
\hline
\end{tabular}

hardening laws [23], as

$\sigma= \begin{cases}E \varepsilon & \varepsilon \leq \sigma^{\mathrm{Y}} / E \\ \sigma^{\mathrm{Y}}\left(\frac{\varepsilon}{\sigma^{\mathrm{Y}} / E}\right)^{N} & \varepsilon>\sigma^{\mathrm{Y}} / E\end{cases}$

where $E$ is the Young's modulus, $N$ is the strain hardening exponent, and $\sigma^{\mathrm{Y}}$ is the yield strength. For the present model, the metallic adherends are assumed to be the high strength steel with these three material properties having the values of $200 \mathrm{GPa}, 0.078$ and 400 MPa [25].

Built upon the bilinear cohesive zone model (CZM), the bondline could be treated as interface between the two metallic adherends, and is modeled with a single layer of four-node cohesive interface elements, which shares nodes with the neighboring elements in the upper and lower metallic adherends. In order to obtain better computational accuracy, the overlap region is densely meshed while sparse mesh is adopted in other regions as shown in Fig. 2b. Furthermore, since the SLJ undergo large displacement and relatively large rotation in the overlap region, especially for the bondline with high separation strength, the geometric nonlinearity has been hence considered here.

\subsection{Model validation}

For the purpose of validating the present theoretical approach for the thickness-dependence cohesive parameters, the overall mechanical response of the SLJ is calculated, with the computational model described above. The features of the cohesive elements applied in modeling the bondline have been presented in Section 2. In this section, a specific commercial epoxy-base adhesive is modeled for the bondline (i.e., Hysol EA 9321, Loctite), with the material properties taken from the previous literature [9] and shown in Table 1.

The calculation is carried out through two steps, which involve the determination of the cohesive parameters, followed by obtaining the mechanical response under external loads. For the first step, the three thickness-dependence important cohesive parameters, namely, the initial stiffness $k_{0}$, total fracture energy $\Gamma$ and separation strength $\hat{\sigma}$, are obtained according to the prediction method mentioned in Section 2.3. Noting that the high strength steel with high elastic modulus is selected as the adherend, the prediction method is hence applicable for the present computational model according to the 


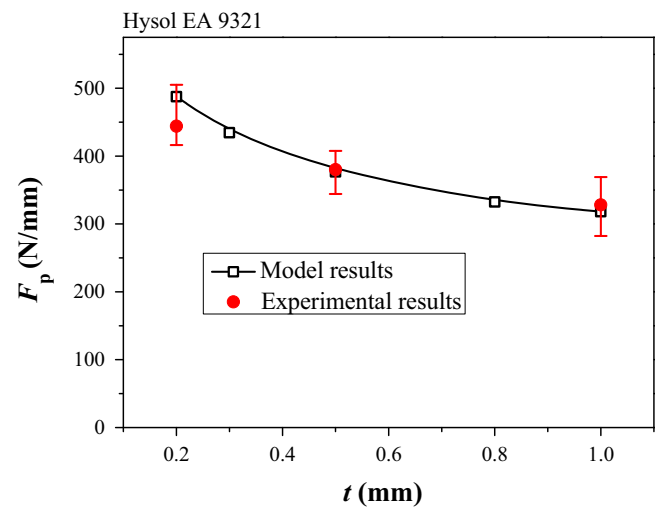

Fig. 3. Peak load plotted as a function of bondline thickness for Hysol EA 9321: comparison between the present model prediction and the experimental results [9].

condition aforementioned in Section 2.3. The cohesive parameters with various thicknesses are considered for validation. For conciseness, the thickness-dependence cohesive parameters for the selected three bondline thicknesses (i.e., $0.1,0.5$ and $1.0 \mathrm{~mm}$ ) are merely presented in Table 2. The parameters for other thicknesses could be obtained as well. It should be noted that the cohesive properties in shear are different from those in normal. It can be observed that the bondline thickness has a significant influence on the cohesive properties. With the decreasing bondline thickness, the separation strength increases gradually, accompanied with the decreasing fracture energy.

By employing the obtained cohesive parameters for the present model, the overall mechanical behavior of the SLJ could be gained. Typical load-displacement curves for the SLJ are shown in Fig. A1 included in Appendix A, considering the bondline thickness of 0.1, 0.5 and $1.0 \mathrm{~mm}$. It should be pointed out the obtained load refers to the load per a unit width of the joint, with the unit of $\mathrm{N} / \mathrm{mm}$, since the plane strain model is adopted. Note that all the loads increase to peak loads and then decline with the increasing displacement. Although the peak loads correspond neither to crack initiation nor to the onset of instability, it has been widely accepted that the overall strength or the load bearing capacity of SLJ could be assessed by the peak values of the load-displacement curves, which are usually called peak loads. Here, the variations of the peak loads for various bondline thicknesses are therefore concerned.

In order to clearly show the influence of bondline thickness on the overall strength of the joints, as presented in Fig. 3, the peak load $F_{p}$ is plotted as a function of the various thicknesses. It should be noted that the selected bondline thicknesses are varied within the range of interval $(0.2,1) \mathrm{mm}$, for the purpose of comparing with the corresponding experimental study which considers the same thickness range [9]. The calculated results depicted in Fig. 3 demonstrate the overall strength of the adhesively bonded structures decreases with the increasing bondline thickness. For checking the feasibility of the present numerical method, the existing experimental results [9] with the adoption of the same adhesives are compared with the present computational predictions. Overall, the present calculated results agree well with those measured. Noting that both the measured and the calculated results depicted in Fig. 3 are original data without any dimensionless processing, thus it can be judged that the accuracy of the present calculated model is satisfactory and the present theoretical approach considering the thickness-dependence cohesive law is convincing.

In order to show the soundness of the present approach to predict the bondline thickness-dependence cohesive parameters, the computational results for the other two types of adhesives mentioned in Ref. [9] are also obtained and compared to the experimental results, which are shown in Appendix B.

The load bearing capacity of the SLJ could be regarded as an overall mechanical property of the structure. The aforementioned a

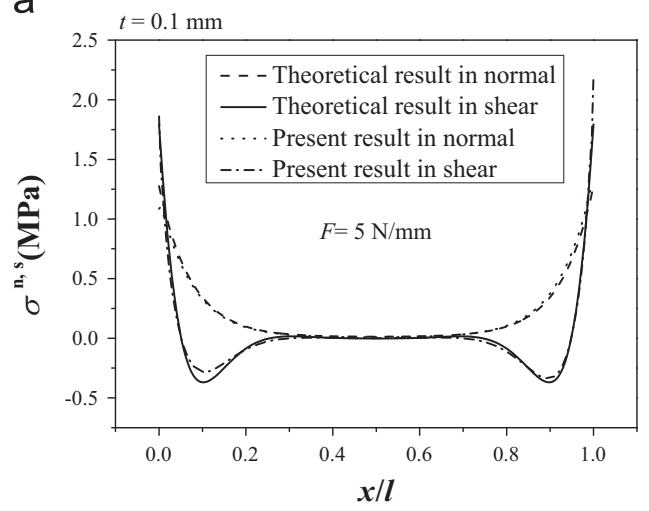

b

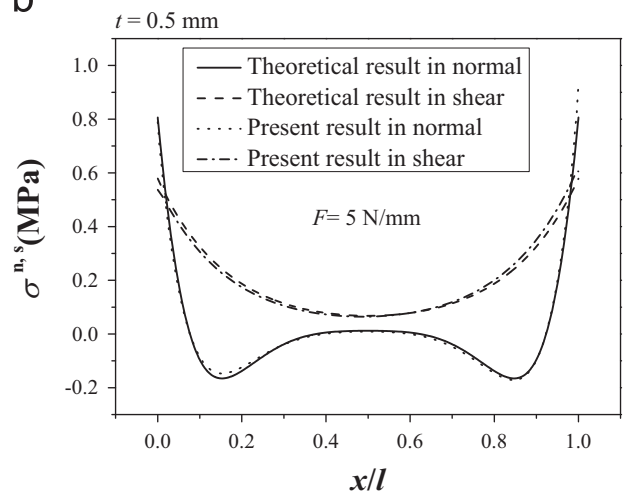

C

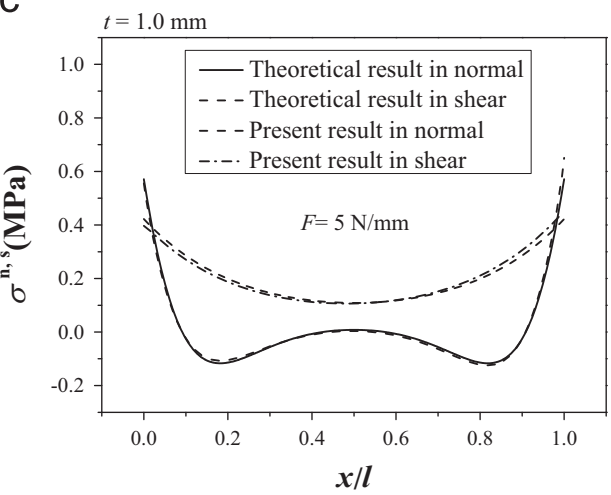

Fig. 4. Stress component distribution in bondline with the thickness of (a) $0.1 \mathrm{~mm}$ (b) $0.5 \mathrm{~mm}$; (c) $1.0 \mathrm{~mm}$ : the present model results in comparison with the classical theoretical solutions [26].

text demonstrates that the present prediction method for the thickness-dependence cohesive parameters is suitable for the overall mechanical assessment. However, it remains questionable whether the stress distribution in the bondline with different thicknesses could be accurately evaluated by the present prediction model. Therefore, the validation for the stress distribution with various bondline thicknesses should be implemented as well. The classical analytical model proposed by Goland and Reissner (i.e., GR model for short) [26] has been widely accepted as an effective method for calculating the stress distribution in the bondline of SLJ. The detailed expressions of the GR model can be found in Appendix C. Here, the GR model results are employed to compare with the present computational results. As shown in Fig. 4, under the selected external load (i.e., $5 \mathrm{~N} / \mathrm{mm}$ ), the present computational results agree perfectly with the GR model results for the considered bondline thickness, which indicates that the present model could properly capture the influence of the thickness on the both normal and shear 
stress distribution in the bondline. Noting the GR model was come out based on the linear elastic theory, while the plastic deformation and damage was not taken into account. In fact, the theoretical method for stress distribution considering the plasticity integrated with damage evolution has been still lacking so far. Thus the validation of the stress distribution is suitable for the elastic situation and the magnitude of load for validation is relatively low.

\section{Results and discussion}

\subsection{Evolution in the bondline extremities}

For adhesively bonded joints, the extremities of the bondline should be concerned due to stress concentration. The stress components near the extremities always present remarkably larger magnitude than those in other locations. Accordingly, the damage onset and crack initiation usually occur in the extremities of the bondline prior to any other locations. In the present investigation, the stress and damage evolution in the extremities are firstly discussed.

Three magnitudes of the bondline thickness are considered based on the calculation with the aforementioned thickness-dependence cohesive parameters. Since the symmetry of the present structure, the stress components in the both extremities are nearly identical, thus only the left extremity of the bondline is considered here. Fig. 5 plots both the stress components and damage variables in the left extremity as a function of the external displacement. In order to facilitate comparison, the dimensionless load-displacement curves (i.e., $F / \hat{\sigma}^{s} l \sim u$ ) of the bonding structure are exhibited together in Fig. 5. The definition of the damage variable $D$ is given by Eq. (6), which describes the damage level of a location point. The damage variable $D$ increases monotonically from 0 (damage initiation) to 1 (complete failure) after the location point goes beyond the damage threshold. Certainly, the damage evolution is an irreversible process. It can be found in Fig. 5 that three cases corresponding to different bondline thicknesses have a notable discrepancy. For the case with the bondline thickness of $0.1 \mathrm{~mm}$, as shown in Fig. 5a, the stress evolution process can be divided into three stages. In stage I, the two stress components increase linearly with the increasing displacement. It can be understood that the shear component increases faster than the normal component since the SLJ is a configuration with the shear deformation dominated. In stage I, the damage variable is unchanged, maintaining the initial value of 0 , which means there is no damage in stage I. Then in stage II, the situation is different, both of the two stress components gradually decline to 0 , obviously, the decline rate of the shear component is relatively large. Meanwhile, the damage variable rise from 0 to 1 in stage II, which suggests it is a damage evolution stage. In stage III, the two stress components in the extremity are both equal to 0 , while the damage variable $D$ keeps at the value of 1 , which denotes the total failure.

Compared with the case shown in Fig. 5a, the case with the thicker bondline exhibits different evolution feature. As illustrated in Fig. $5 \mathrm{c}$ denoting the bondline of $1.0 \mathrm{~mm}$, four stages can be found in the curves. Among them, stage I, II and the final stage are similar to the situation shown in Fig. 5a. It should be noted that the normal stress component changes negligibly in stage II while the shear stress component declines obviously. Furthermore, the other significant feature in Fig. $5 \mathrm{c}$ is the existence of the second damage evolution, namely, stage III, in which the both stress components decline drastically. Consequently, the damage variable rise drastically. And the change rates of both the stress components and damage variable are distinctly larger than those in stage II.

For the case with the bondline of $0.5 \mathrm{~mm}$ depicted in Fig. 5b, the curves exhibit the feature between the two aforementioned cases. The most significant discrepancy comparing with the other cases is that stage III in Fig. 5b is not notable, though could be observed.
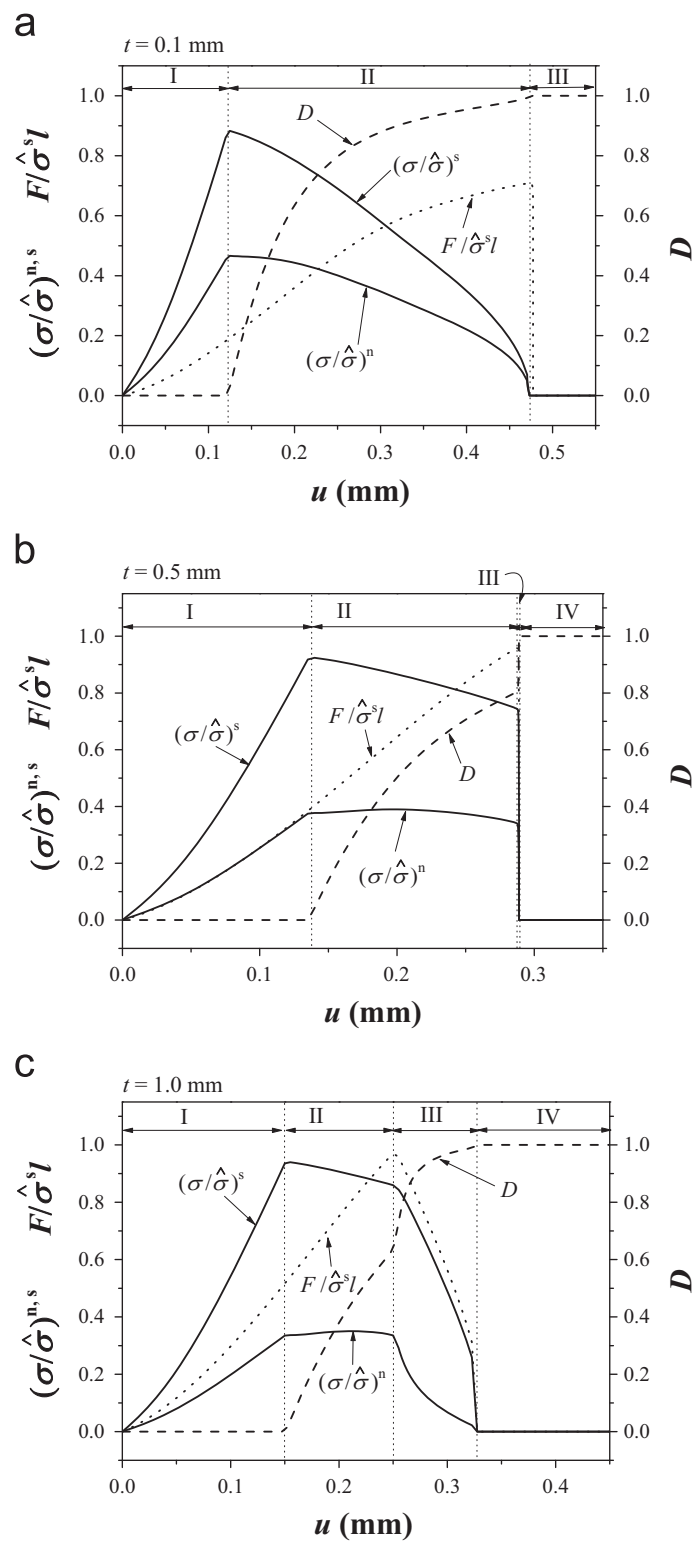

Fig. 5. Stress components and damage variable evolution in the extremities of the bondline with the thickness of (a) $0.1 \mathrm{~mm}$; (b) $0.5 \mathrm{~mm}$; (c) $1.0 \mathrm{~mm}$, and the loading curve is exhibited together for comparison.

In stage III, the stress components and damage variable change suddenly. As a result, the range of stage III appears extremely small.

As mentioned in the above text, the extremities of the bondline are the critical points due to the existence of stress concentration. Thus some researchers have assessed the overall strength of the bonding structures by examining the stress state of the extremities $[5,27]$. However, it is a conservative method for the safety assessment of the structures. As shown in Fig. 5(a-c), the maximum magnitudes of both shear and normal components are reached meanwhile, when the damage initiation occurs in the extremity. It is just the beginning of stage II, which is not the time that the external load reaches its peak value. Actually, at the beginning of stage II, the external loads for the bondline of $0.1,0.5$ and $1.0 \mathrm{~mm}$ are merely $0.25 F_{p}, 0.41 F_{p}$ and $0.56 F_{p}$, respectively, where $F_{p}$ denotes their respective peak load. Accordingly, assessment of overall strength by merely checking the maximum stress in the bondline extremities would result in a significant underestimate, which is larger as the bondline thickness is decreased. However, it is quite interesting to observe the peak load 
a

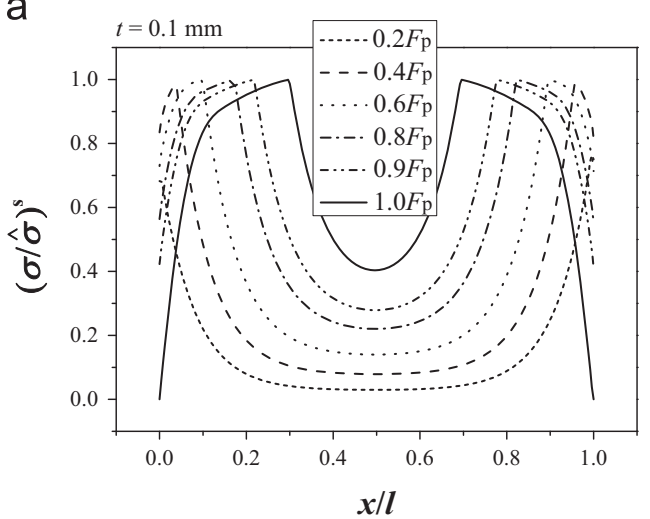

C

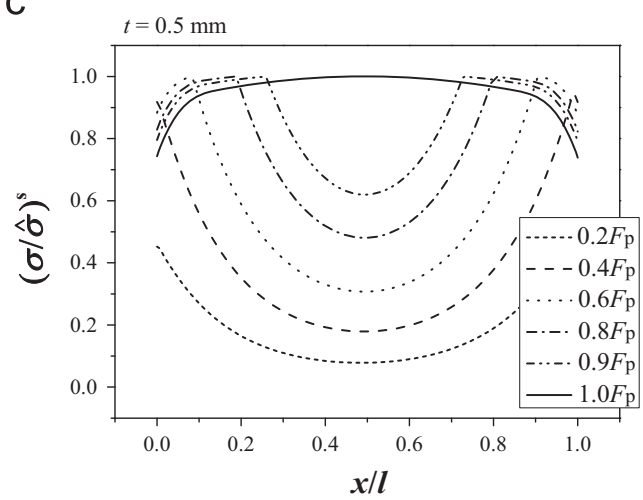

e

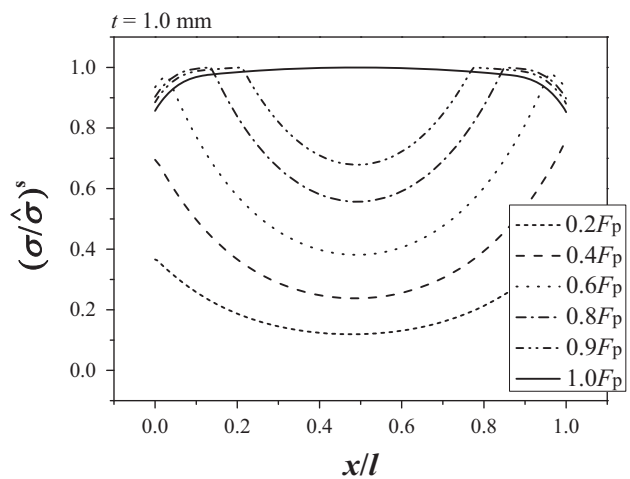

b

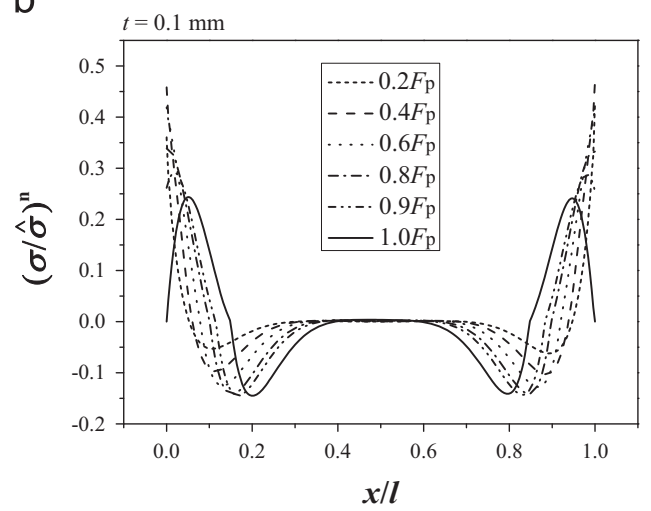

d

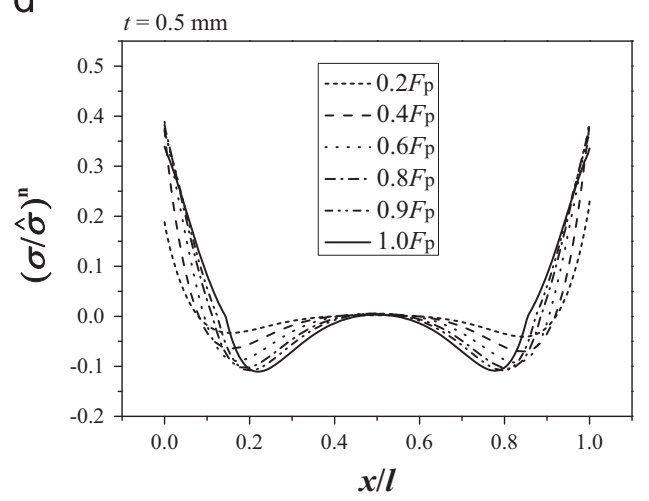

f

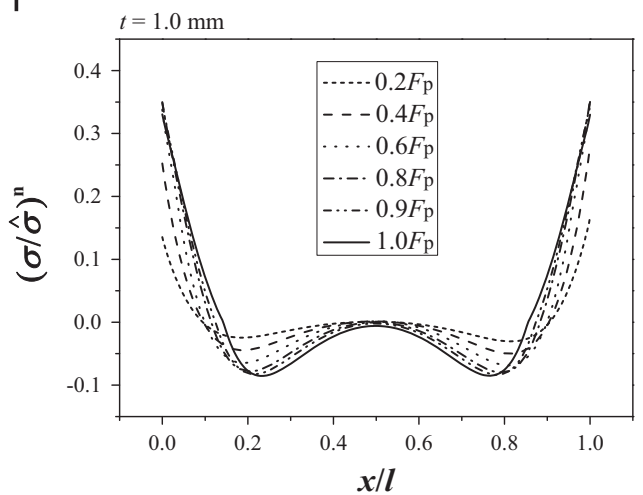

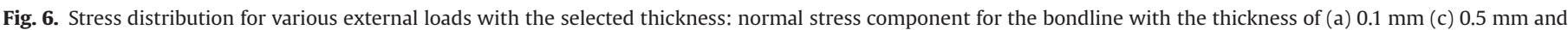
(e) $1.0 \mathrm{~mm}$; shear stress component for the bondline with the thickness of (b) $0.1 \mathrm{~mm}$ (d) $0.5 \mathrm{~mm}$ and (f) $1.0 \mathrm{~mm}$.

is reached at the end of stage II for the three bondline thickness. As mentioned in the above text, stage II is the first damage evolution stage, which is the sole damage evolution stage for relatively thinner bondline (e.g., $0.1 \mathrm{~mm}$ ). The second stage of damage evolution would exist for relatively thicker bondline (e.g., $1.0 \mathrm{~mm}$ ).

In a word, the load at the end of stage II would be more appropriate for assessing the overall strength than the beginning of stage II, based on the consideration of fully exploring the potential load-bearing capacity of the bonding structures.

\subsection{Evolution of stress distribution}

In this section, the evolution of stress distributions considering the influence of damage would be presented. Fig. 6 plots the stress distribution in the bondline for the various external loads, considering three magnitudes of the bondline thickness. As same as Section 4.1, $F_{p}$ in each figure denotes the respective peak load, which has different magnitudes due to different bondline thicknesses. In order to facilitate comparison, a serial of same relative external loads (i.e., $F / F_{p}$ ) are selected. It can be clearly found the combined effect of both the bondline thickness and the damage evolution on the stress distribution. Taking Fig. 6a for example, when the external load is relatively small (e.g., $F=0.2 F_{p}$ ), the deformation and stress state of the bondline are still in the linear elastic range, without any damage. The peak value of the stress component in the bondline still lies in the extremities. However, when the external load is relatively large (e.g., $F \geq 0.4 F_{p}$ ), the situation changes. The location corresponding to the shear peak stress gradually moves towards the center of the bondline. Similarly, the cases with the bondline thickness of 0.5 and $1.0 \mathrm{~mm}$ have the same tendency. It should be noted that the shear stress distribution for the respective peak load exhibits different features. With the increasing bondline thickness, the shear stress distribution becomes uniform along the bondline. Particularly for the bondline of $1.0 \mathrm{~mm}$, the shear stress distribution curve for the peak load appears nearly a straight line except for the region near the extremities.

In contrast with the shear stress component, the situation for the normal stress component is totally different. Generally, the normal stress levels corresponding to the three bondline thicknesses are 
a

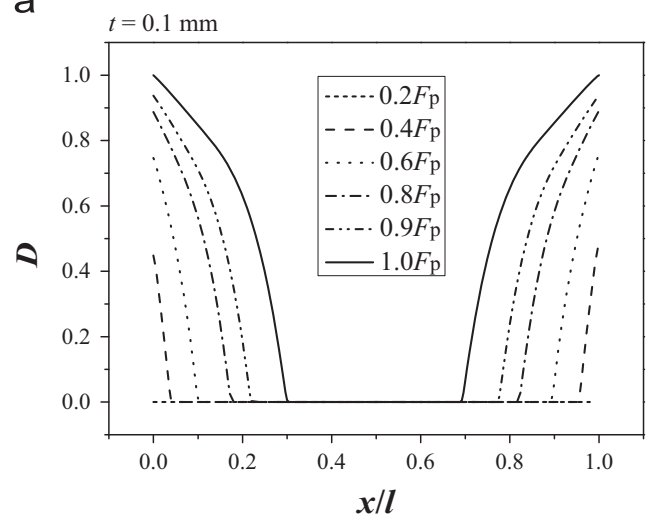

b

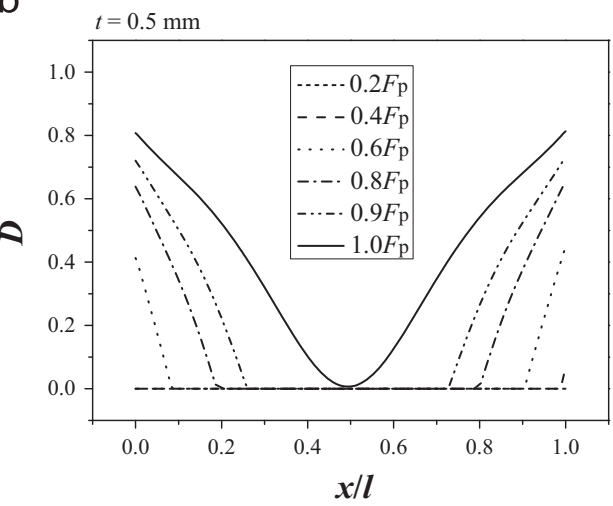

C

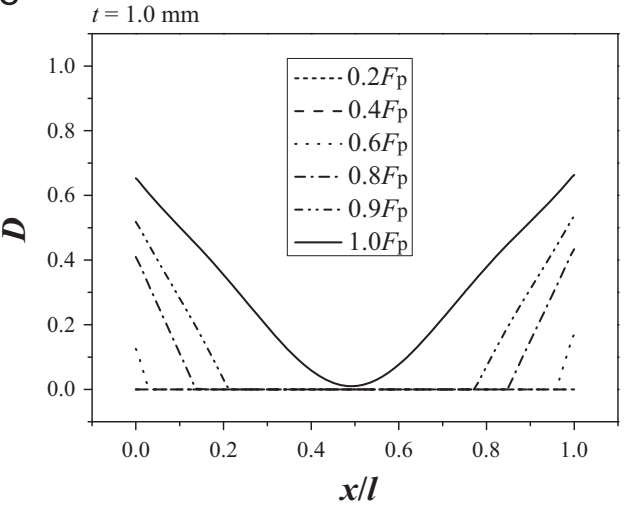

Fig. 7. Damage variable distribution for various external loads in the bondline with the thickness of (a) $0.1 \mathrm{~mm}$; (b) $0.5 \mathrm{~mm}$; (c) $1.0 \mathrm{~mm}$.

low, which could be also understood by the SLJ configuration dominated by shear deformation. Consequently, as the load is increased, the overall shape of the normal stress curves changes slightly, with the magnitude of the normal stress increased visibly.

It can be found in Fig. 6 that the peak stress components do not always locate in the extremities of the bondline, which cannot be predicted by elastic-plastic theories. With the increasing load, the stress concentration of the extremities would be weakened gradually since the damage evolution in the extremities is intensive. Moreover, the peak of the stress curves, especially involving the shear component, would move towards the center of the bondline.

\subsection{Evolution of damage distribution}

As pointed out by the aforementioned text, the variation of the stress distribution has a close relationship with the damage evolution, thus it is necessary to exhibit the evolution of the damage variable distribution under the increasing external load. In order to a

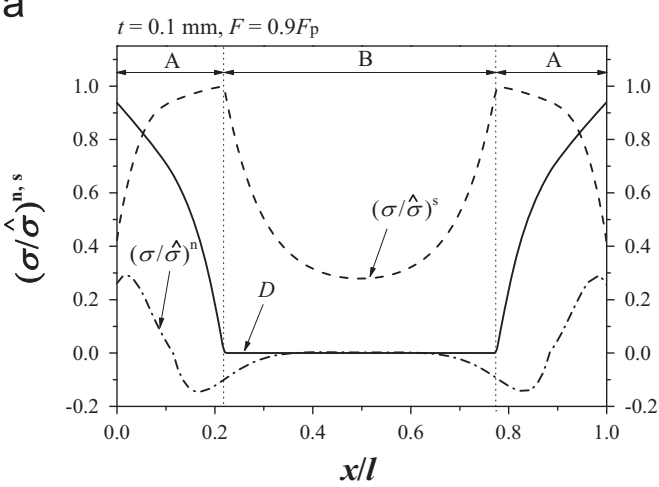

b

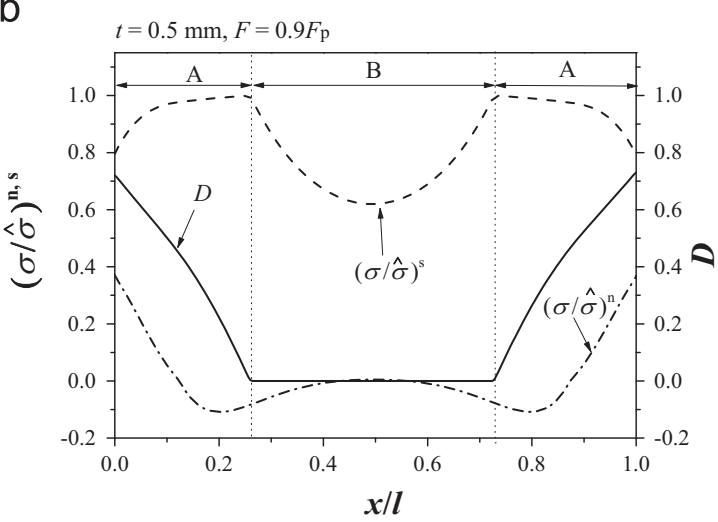

C

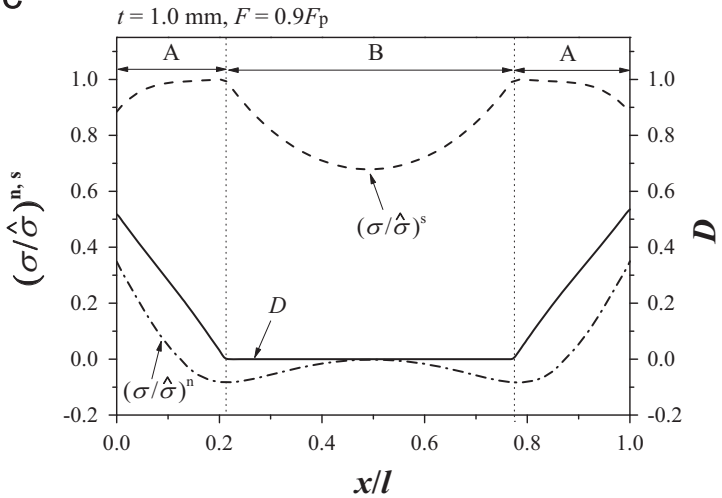

Fig. 8. Distribution of the stress components together with the damage variable for the load of $0.9 F_{p}$, with the bondline thickness of (a) $0.1 \mathrm{~mm}$; (b) $0.5 \mathrm{~mm}$; (c) $1.0 \mathrm{~mm}$.

compare with the aforementioned stress distribution evolution analyses, the same serials of loads are selected in this section.

Fig. 7 plots the damage variable distributions in the bondline under the increasing load, considering the same three magnitudes of the bondline thickness. In general, the damage level near the extremities is high, with the magnitude of damage variables getting increasingly low towards the center of the bondline. Moreover, it is obvious to find out the discrepancy between the figures for various bondline thicknesses. For the relatively thinner bondline (i.e., $t=0.1 \mathrm{~mm}$ ), the range of the damage variable spans from 0 to 1 when the peak loads (i.e., $1.0 F_{p}$ ) is reached, while the other ranges of damage variable at other loads are also large except for that at $0.2 F_{p}$. However, the span of damage zone in the bondline is not wide, which can be demonstrated that the damage variable of the central area still retain at 0 even at the peak load. The undamaged central area is approximately $1 / 3$ of the bondline. By contrast, when the bondline is comparatively thicker, the situation is distinctly different. As shown in Fig. 7b and c, nearly all the bondline are damaged except 


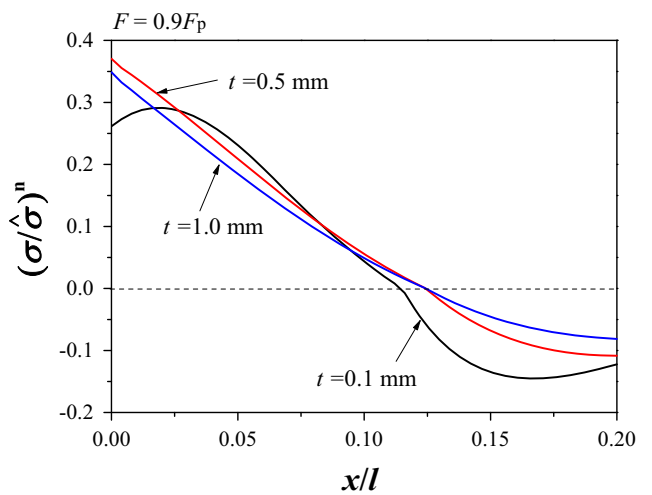

Fig. 9. Normal stress distribution in the bondline within the range close to the left extremity.

for the midpoint. And the other significant discrepancy is that the range of damage variable for the thicker bondline is relatively narrower. In other words, even for the case under the peak load, the maximum value of damage variables is getting smaller as the bondline is increased. For example, the maximum damage variable is only 0.653 for the case with the bondline of $1.0 \mathrm{~mm}$, while the maximum damage variable can reach up to 1.0 for that of $0.1 \mathrm{~mm}$. which can be explained from the view of capacity of plastic dissipation due to the bondline thickness. As mentioned in Section 2.3 , the intrinsic cohesive energy (or fracture energy) $\Gamma_{0}$ for various bondline is same, however, the plastic dissipation energy $\Gamma_{p}$ for the thicker bondline is larger. As a result, the softened stage of the bondline with the thicker bondline is comparatively longer and the damage variable is comparatively smaller under the peak load.

Furthermore, as mentioned above, the damage evolution is linked closely to the stress distribution. Some visible evidences should be presented. It would be clearer by showing both the stress distribution and the damage variable curves together. Fig. 8 illustrates the distribution of the stress components together with the damage variable under the selected load of $0.9 F_{p}$. Although various bondline thicknesses are taken into account, the overall shape feature of the curves is so similar among Fig. 8a, b and c. It is notable to observe the three segments in the curves, namely, two segments labeled with A and one segment labeled with $B$, which denote the damaged and undamaged zones, respectively. It is explicit to observe that the boundaries between Segment A and B are exactly the turning points of the shear stress curves, which can be also observed in the results of the cases under other loads.

In contrast with the curves for the shear stress component, the shape of the curves for the normal stress component does not change remarkably in the damaged areas (i.e., Segment A). In order to clearly present the normal stress distribution in the damaged areas, the curves for the normal stress are manifested by considering the bondline within the range of $(0 \sim 0.2 l)$. Due to the symmetry, only the situation for the left side in the curves is presented. It can be found a dashed horizontal line in Fig. 9, with the vertical coordinate of 0 , which separates the regions of the positive and negative normal stress. The stress curves in the region below the dashed line refers to compressive stress, while that above the dashed lines refers to tensile stress. It should be noted that the area in bondline selected in Fig. 9 (i.e., $0 \sim 0.2 l$ ) is included in the damaged areas in Fig. 8. However, there is another important postulate should be reminded here, namely, compressive stress cannot contribute to both the damage initiation and the damage evolution, as depicted in Eqs. (2) and (6). Thus the smooth transition could be observed in the normal stress curves across the boundary between the damaged and undamaged regions, since the magnitudes of the normal stress curves near the boundary are both negative. As shown in Fig. 9, the curves exhibit turning points where they go across the dashed line. The turning point is more distinct when the bondline thickness is relatively smaller. As described above, the dashed line is the boundary between the tensile and compressive normal stresses, in other words, it can be also regarded as the boundary between the damaged and undamaged region for the normal stress. Thus it can be easily understood the existence of the turning point. Nevertheless, it is worth mentioning that the effect of the turning points in normal stress curves in Fig. 9 is not as significant as those in shear stress curves in Fig. 8. It can be also comprehended that the damage initiation and evolution are mainly contributed by the shear deformation and shear stress for the configuration of SLJ. Compared with the shear stress component, the overall normal stress level is very low, thus the damage affects the shear stress component more strongly than the normal component. Similarly, since the overall stress levels for the thinner bondline are larger, including the normal and shear components, the stress curves for the thinner bondline are more susceptible to the damage. Thus the changes in the stress curves near the turning points are more remarkable for the thinner bondline as shown in Figs. 8 and 9.

\subsection{Further discussion}

In the present investigation, only one specified adhesive (i.e., Hysol EA 9321) is taken into account here. It is worth noting that a dimensionless parameter $\eta$, (i.e., toughness ratio) is introduced in the prediction method regarding the thickness-dependence bondline. As described in Section 2.3, $\eta$ is an intrinsic parameter of adhesive materials despite of their size and shape. Thus the magnitude of $\eta$ in the bondline with various thicknesses is unique for every specific adhesive. However, obviously, different from the intrinsic material toughness, the bondline toughness is varied due to the various thicknesses, which needs to be further explained reasonably. Recently, the cohesive length scale $\zeta$, with the definition depicted in Eq. (16), has been introduced and used in the issues regarding the damage and failure of the bonding structures .

$\zeta=\frac{E \Gamma}{\hat{\sigma}^{2}}$

where $E$ is the Young's modulus of substrate materials adjacent to the crack plane, $\Gamma$ and $\hat{\sigma}$ are the cohesive parameters with the same signification in Section 2.1. The virtue of cohesive length scale $\zeta$ is that it brings together the fracture properties of a bondline in a way of one single parameter which can be used instead for the description and further classification of the bondline. Considering the condition of $t<t_{c}$ and combining Eq. (9), (13) and (14), then Eq. (16) can be rewritten as,

$\zeta=\frac{k_{c} \Gamma_{0}(1+\eta) t}{\sigma_{f}^{2}} \quad\left(t<t_{c}\right)$

For the bilinear cohesive zone model employed in this study, $\Gamma_{0}$ could be regarded as the intrinsic work of fracture associated with the embedded cohesive zone. As depicted in Fig. 1, the following relations could be easily found,

$\Gamma_{0}=\frac{1}{2} \sigma_{f} \delta_{c}$

$k_{c}=\frac{\sigma_{f}}{\delta_{c}}$

Thus, $\zeta$ could be further simplified into the following form,

$\zeta=\frac{1}{2}(1+\eta) t \quad\left(t<t_{c}\right)$

Considering the condition of $t \geq t_{c}, \zeta$ degrades to a constant,

$\zeta=\frac{1}{2}(1+\eta) t_{c} \quad\left(t \geq t_{c}\right)$ 


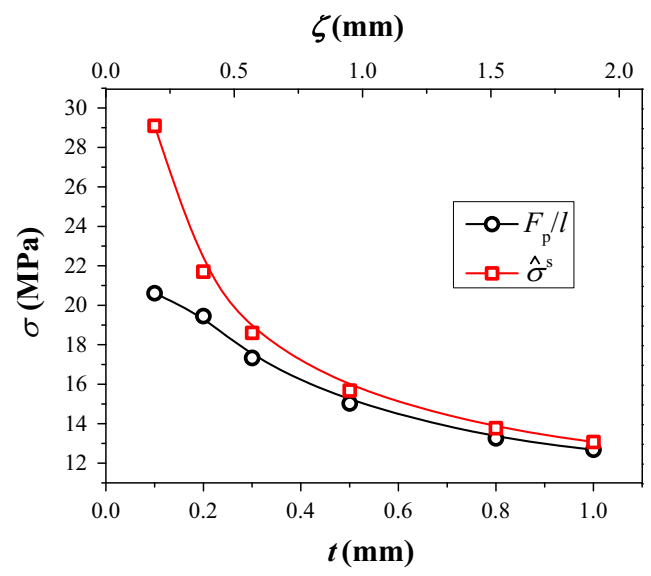

Fig. 10. Average shear strength $\left(F_{p} / l\right)$ approaches gradually to the shear cohesive strength $\hat{\sigma}^{s}$ as the bondline thickness and cohesive length scale are increased.

The previous researches have been indicated that $\zeta$ can be used to judge whether the failure of bondline is brittle or ductile $[28,30]$. In the present investigation, obviously, the case for the thinner bondline appears brittle failure while the case for thicker bondline appears ductile failure although the same type of adhesive is selected, as shown in Fig. A1, which can be understood by Eq. (20).

It is so explicit that $\zeta$ is determined by two parameters, namely, toughness ratio $\eta$ and bondline thickness $t$. Thus the toughness of the bondline could be increased by either selecting high-toughness adhesive or increasing the bondline thickness, each of which has the equivalent effect. However, when the bondline thickness is larger than the critical value, the value of $\zeta$ would not be changed any more. The value of $\eta$ for the present selected adhesive (i.e., Hysol EA 9321) is 2.8, taken from Ref. [12]. And the present bondline thickness range (i.e., $t \leq 1.0 \mathrm{~mm}$ ) is smaller than the critical thickness $t_{c}$, Thus the $\zeta$ is merely the function of bondline thickness $t$, namely, $\zeta=1.9 t$.

In other aspect, as mentioned in Section 3.2, numerous literatures and test standards have regarded the ratio between the peak loads and overlap area as the assessment for the shear bondline strength. The previous researches have reported that the ratio increases approaching to a steady value, with the decreasing overlap length (i.e., bonding area), which suggests that the strength assessment is well suitable in short lap joints [29] In the present study, the ratio $F_{p} / l$ and shear separation strength $\hat{\sigma}^{s}$ are plotted together as the function of bondline thickness $t$ and cohesive length scale $\zeta$. As illustrated in Fig. 10 , the shear strength obtained by $F_{p} / l$ approaches to the shear separation strength $\hat{\sigma}^{s}$ as the bondline thickness $t$ (or, cohesive length scale $\zeta$ ) is increased. Anyfantis [29] has claimed that the failure analysis of bondlines characterized by relatively high cohesive length scale $\zeta$ should be done with stress-based approaches, because the debonding in such cases is a cohesive strength driven procedure. Since the strength assessment by the ratio $F_{p} / l$ is a method with the stressbased approach, the result depicted in Fig. 10 can be also explained by the conclusion of Ref. [29]. In a word, the strength assessment method based on $F_{p} / l$ is appropriate for the bondline with high cohesive length scale, which could be obtained by either selecting hightoughness adhesive or increasing the bondline thickness.

\section{Conclusions}

In summary, the influence of the thickness on the damage and stress evolution in the bondlines of metallic single lap joints are investigated, with the cohesive zone model employed to equivalently simulate the bondline with various thicknesses. A prediction approach is employed to determine the cohesive parameters for

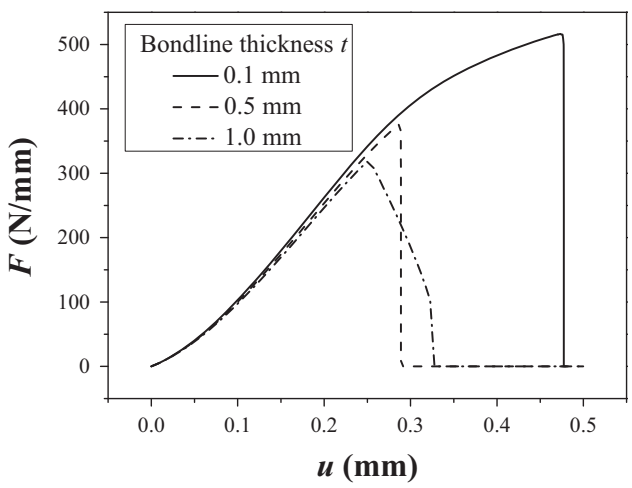

Fig. A1. Typical load-displacement curves of SLJ with the selected bondline thickness.

the present model when the bondline thickness is varied. And then a FEM model is built to explore the bondline thickness-dependence damage and stress distribution evolution in the bondline, involving the situations of the extremities and the whole bondline. The results show that the damage and stress evolutions are mutually influential processes, both of which are affected by the bondline thickness. For the extremities in the bondline, as the bondline thickness is increased, the second damage evolution stage with rapid degradation would exist and develop. Furthermore, the shear peak stress locations in the stress distribution curves would move towards the center of the bondline due to the evolution of the damage zone which initiates from the extremities. The boundaries between the damaged area and the undamaged area are exactly coincident with the turning points of the shear stress distribution curve. Moreover, for the SLJ under the peak load, the shear stress distribution is getting more uniform while the overall damage level is getting smaller as the bondline thickness is increased. Finally, the effect of the bondline thickness on the accuracy of the overall strength assessment could be evaluated by the cohesive length scale.

\section{Acknowledgments}

This work was supported by the National Basic Research Program of China (No. 2011CB605506), and by the Project funded by China Postdoctoral Science Foundation (No. 2013M540033). The first author (WX) would like to thank the helpful advice of Prof. Wei Yueguang from Institute of Mechanics, Chinese Academy of Sciences.

\section{Appendix A}

In Section 3.2, the peak loads are obtained by the underlying load-displacement relations of SLJ subjected to tensile loads. Fig. A1 shows the typical load-displacement curves of SLJ with the selected thickness.

\section{Appendix B}

By the prediction approach of the bondline thickness-dependence cohesive parameters, the peak loads of the adhesive joints with the other two types of adhesives (i.e., Hysol EA 9361 and AV138/HV998) can be also obtained and compared to the experimental results, which are shown in Fig. A2. Overall, the present model results agree with those experimental results as well for these two types of adhesives. 
a

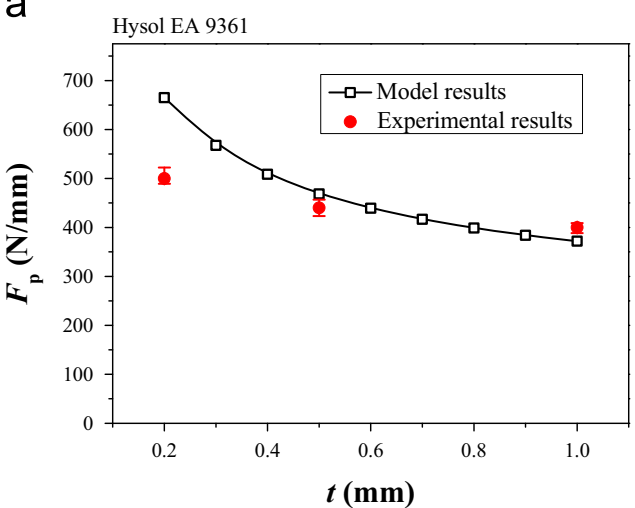

b

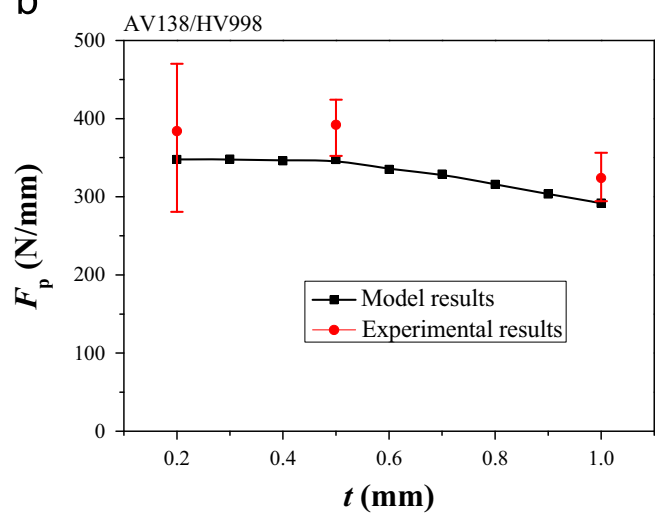

Fig. A2. Peak load plotted as a function of bondline thickness for (a) Hysol EA 9361 and (b) AV138/HV998: comparison between the present model prediction and the experimental results. [9].

\section{Appendix C}

The Goland and Reissner model [26] was proposed to calculate the stress distribution of bondlines in the configuration of single lap joints (SLJ) subjected to tensile loads. The expression for adhesive shear stress is

$\tau=-\frac{1}{8} \frac{F}{c}\left\{\frac{\beta c}{t^{\prime}}(1+3 k) \frac{\cosh \left(\left(\beta c / t^{\prime}\right)(x / c)\right)}{\sinh \left(\beta c / t^{\prime}\right)}+3(1-k)\right\}$

where $F$ is the applied tensile load per unit width, $c$ half of the overlap length (i.e., $c=0.5 l$ ), $t^{\prime}$ the adherend thickness, $t$ the bondline thickness.

$\beta^{2}=8 \frac{G_{\mathrm{a}}}{E} \frac{t^{\prime}}{t}$

$k=\frac{\cosh \left(u_{2} c\right)}{\cosh \left(u_{2} c\right)+2 \sqrt{2} \sinh \left(u_{2} c\right)}$

$u_{2}=\sqrt{\frac{3\left(1-\nu^{2}\right)}{2}} \frac{1}{t^{\prime}} \sqrt{\frac{F}{t^{\prime} E}}$

where $G_{a}$ is the adhesive shear modulus and $E$ is the Young's modulus of adherend.

And the expression for the normal stress is

$\sigma=-\frac{1}{\Psi} \frac{F t^{\prime}}{c}\left[\begin{array}{l}\left(R_{2} \lambda^{2} \frac{k}{2}+\lambda k^{\prime} \cosh (\lambda) \cos (\lambda)\right) \cosh \left(\frac{\lambda x}{c}\right) \cos \left(\frac{\lambda x}{c}\right)+ \\ \left(R_{1} \lambda^{2} \frac{k}{2}+\lambda k^{\prime} \sinh (\lambda) \sin (\lambda)\right) \sinh \left(\frac{\lambda x}{c}\right) \sin \left(\frac{\lambda x}{c}\right)\end{array}\right]$ where

$\lambda=\gamma \frac{c}{t^{\prime}}$

$\gamma^{4}=6 \frac{E_{a}}{E} \frac{t^{\prime}}{t}$

where $E_{a}$ is the adhesive Young's modulus,

$k^{\prime}=\frac{k c}{t^{\prime}} \sqrt{3\left(1-\nu^{2}\right) \frac{F}{t^{\prime} E}}$

$R_{1}=\cosh (\lambda) \sin (\lambda)+\sinh (\lambda) \cos (\lambda)$

$R_{2}=\sinh (\lambda) \cos (\lambda)-\cosh (\lambda) \sin (\lambda)$

$\Psi=\frac{1}{2}(\sin (2 \lambda)+\sinh (2 \lambda))$

It should be noted that the origin of the longitudinal coordinate $x$ is the middle of the overlap for the Goland and Reissner model.

\section{References}

[1] He XC. A review of finite element analysis of adhesively bonded joints. Int J Adhes Adhes 2011:31:248-64.

[2] da Silva LFM, Ochsner A. Modeling of adhesively bonded joints. Berlin: Springer Verlag; 2008.

[3] da Silva LFM, das Neves PJC, Adams RD, Spelt JK. Analytical models of adhesively bonded joints-Part I: literature survey. Int J Adhes Adhes 2009;29:319-30.

[4] da Silva LFM, das Neves PC, Adams RD, Wang A, Spelt JK. Analytical models of adhesively bonded joints-Part II: comparative study. Int J Adhes Adhes 2009;29:331-41.

[5] Weißgraeber P, Becker W. Finite Fracture Mechanics model for mixed mode fracture in adhesive joints. Int. J. Solids Struct. 2013:50:2383-94.

[6] You M, Yan ZM, Zheng XL, Yu HZ, Li Z. A numerical and experimental study of adhesively bonded aluminium single lap joints with an inner chamfer on the adherends. Int J Adhes Adhes 2008;28:71-6.

[7] Ernesto Mendoza-Navarro L, Diaz-Diaz A, Castañeda-Balderas R, Hunkeler S, Noret R. Interfacial failure in adhesive joints: experiments and predictions. Int J Adhes Adhes 2013;44:36-47.

[8] Crocombe AD. Global yielding as a failure criterion for bonded joints. Int J Adhes Adhes 1989;9:145-53.

[9] da Silva LFM, TNSS Rodrigues, Figueiredo MAV, de Moura MFSF, Chousal JAG. Effect of adhesive type and thickness on the lap shear strength. J. Adhes. 2006; 82:1091-115.

[10] Ji GF, Ouyang ZY, Li GQ Ibekwe S, Pang SS. Effects of adhesive thickness on global and local Mode-I interfacial fracture of bonded joints. Int J Solids Struct 2010:47:2445-58.

[11] Liao L, Huang C, Sawa T. Effect of adhesive thickness, adhesive type and scarf angle on the mechanical properties of scarf adhesive joints. Int J Solids Struct 2013;50:4333-40.

[12] Xu W, Wei Y. Influence of adhesive thickness on local interface fracture and overall strength of metallic adhesive bonding structures. Int J Adhes Adhes 2013;40:158-67.

[13] Adnan A, Sun CT. Effect of adhesive thickness on joint strength: a molecular dynamics perspective. J Adhes 2008;84:401-20.

[14] Moradi A, Leguillon D, Carrère N. Influence of the adhesive thickness on a debonding - an asymptotic model. Eng Fract Mech 2013;114:55-68.

[15] Hell S, Weißgraeber P, Felger J, Becker W. A coupled stress and energy criterion for the assessment of crack initiation in single lap joints: a numerical approach. Eng Fract Mech 2014;117:112-26.

[16] Chandra N, Li H, Shet C, Ghonem H. Some issues in the application of cohesive zone models for metal-ceramic interfaces. Int J Solids Struct 2002;39:2827-55.

[17] Hui CY, Ruina A, Long R, Jagota A. Cohesive zone models and fracture. J Adhes 2011:87:1-52.

[18] Freund LB, Suresh S. Thin film materials: stress, defect formation and surface evolution. Cambridge: Cambridge University Press; 2003.

[19] Chow CL, Lu TJ. On evolution laws of anisotropic damage. Eng Fract Mech 1989;34:679-701.

[20] Balzani C, Wagner W. An interface element for the simulation of delamination in unidirectional fiber-reinforced composite laminates. Eng Fract Mech $2008 \cdot 75 \cdot 2597-615$

[21] de Moura MFSF, RDSG Campilho, JPM. Goncalves. Mixed-mode cohesive damage model applied to the simulation of the mechanical behaviour of laminated composite adhesive joints. J Adhes Sci Technol 2009;23:1477-91.

[22] Hogberg JL. Mixed mode cohesive law. Int J Fract 2006;141:549-59. 
[23] Pardoen T, Ferracin T, Landis CM, Delannay F. Constraint effects in adhesive joint fracture. J Mech Phys Solids 2005;53:1951-83.

[24] Wei YG, Zhao HF. Peeling experiments of ductile thin films along ceramic substrates - critical assessment of analytical models. Int J Solids Struct 2008;45:3779-92.

[25] Xu W, Wei Y. Assessments for impact of adhesive properties: modeling strength of metallic single lap joints. J Adhes Sci Technol 2013;27:9-29.

[26] Goland M, Reissner E. The stresses in cemented joints. J Appl Mech 1944;66: A17-A27.
[27] Goglio L, Rossetto M, Dragoni E. Design of adhesive joints based on peak elastic stresses. Int J Adhes Adhes 2008;28:427-35.

[28] Sills RB, Thouless MD. Cohesive-length scales for damage and toughening mechanisms. Int J Solids Struct 2015;55:32-43.

[29] Anyfantis KN. On the failure analysis of bondlines: stress or energy based fracture criteria? Eng Fract Mech 2014;126:108-25.

[30] Sills RB, Thouless MD. The effect of cohesive-law parameters on mixed-mode fracture. Eng Fract Mech 2013;109:353-68. 